\title{
On the Dispersive Ordering and Applications
}

\author{
Tran Loc Hung ${ }^{1}$ and Nguyen Van Son ${ }^{2}$ \\ ${ }^{1}$ Department of Mathematics and Statistics, Faculty of Basic Science, University of Finance-Marketing (UFM), \\ 306 Nguyen Trong Tuyen Street, Tan Binh District, Ho Chi Minh City, Vietnam \\ ${ }^{2}$ Department of Mathematics, Hue University of Science, 77 Nguyen Hue Street, Hue City, Vietnam
}

Correspondence should be addressed to Nguyen Van Son; son.nv2001@gmail.com

Received 20 May 2013; Accepted 24 July 2013

Academic Editors: M. Brünig and Q. Song

Copyright (C) 2013 T. L. Hung and N. V. Son. This is an open access article distributed under the Creative Commons Attribution License, which permits unrestricted use, distribution, and reproduction in any medium, provided the original work is properly cited.

The purpose of this paper is to present some results related to the dispersive ordering of probability distributions via dispersion functions of the $\mathscr{L}^{1}$-random variables. A new approach to the Laws of Large Numbers in $\mathscr{L}^{1}$-norm can be applied via received results. A new concept on minimum-dispersive unbiased estimator is considered, too.

\section{Introduction}

Let $X$ be a random variable defined on a probability space $(\Omega, \mathfrak{A}, P)$, with distribution $F_{X}$ and mean $E(X)$. A random variable $X$ is called to belong to class $\mathscr{L}^{1}$, if its mean is finite. From now on, $D_{X}(u)$ denotes the dispersion function of $\mathscr{L}^{1}$ random variable $X$ at a point $u \in(-\infty,+\infty)$, defined as follows:

$$
\begin{array}{r}
D_{X}(u)=E|X-u|=\int_{-\infty}^{+\infty}|x-u| d F_{X}(x), \\
u \in(-\infty,+\infty)
\end{array}
$$

It is to be noticed that the dispersion function in (1) also is known as the absolute deviation function of $X$ at a point $u \in(-\infty,+\infty)$ (see [1] for more details). Up to the present, some results related to dispersion functions in term of (1) have been investigated by Muñoz-Perez and Sanchez-Gomez $[2,3]$, Pham-Gia and Hung [1], and Hung and Son [4]. Also note that the dispersive functions and stochastic ordering have been considered in various papers and they are effective tools in many areas of probability and statistics. Such areas include reliability theory, queuing theory, survival analysis, biology, economics, insurance, actuarial science, operations research, and management science (we refer to $[5,6]$ for a complete treatment of the problem).

It is worth pointing out that the dispersion function $D_{X}(u)$ of $X$ at a point $u \in(-\infty,+\infty)$ has attracted much attention as a dispersion measure of $X$ in $\mathscr{L}^{1}$-norm and it can be considered as a generalization of the mean absolute deviation $\delta_{1}(\mu):=E|X-\mu|$ and the median absolute deviation $\delta_{2}(X):=E|X-M d|$ of a random variable $X$ when $\mu$ and $M d$ exist and are unique; here and throughout this paper $\mu$ and $M d$ denote the mean and median of random variable $X$, respectively. The mean absolute deviation and median absolute deviation that play particular roles in Applied Statistics and Economics have been investigated by Pham-Gia et al. (we refer the reader to [1,7-9] for deeper discussions).

The dispersion function as was stated above is convex and almost everywhere differentiable, and its derivative has most a countable numbers of discontinuity points (see for instance [2, 3]). Lately, some interesting results concerning the connections of the weak convergence of a sequence of $\mathscr{L}^{1}$ random variables with the convergence of their corresponding dispersion functions have been investigated by Hung and Son (see [4] for more details). Thus, the dispersion function $D_{X}(u)$ of random variable $X$ at a point $u \in(-\infty,+\infty)$ has attracted much attention as a dispersion measure of a random variable $X$ in various problems related to limit theorems of probability theory, applied statistics, and economics.

The main aim of this paper is to present some results related to the dispersive ordering of probability distributions via dispersion functions of the $\mathscr{L}^{1}$-random variables. The received results are extensions of authors studies in [4], and they are showing a new approach to the Laws of Large Numbers in $\mathscr{L}^{1}$-norm. 
The organization of this paper is as follows. In Section 2 we will recall the main properties of the dispersion functions that will play fundamental roles in the study of next section. For more details about the proofs of results in this section, we refer the reader to Muñoz-Perez and Sanchez-Gomez [2, 3 ] and Hung and Son [4]. The last section gives some main results on dispersive ordering of probability distributions via dispersion functions and applications.

\section{Preliminary Results}

Some properties of the dispersion functions have been investigated so far; they can be listed as follows. For more details we refer the reader to [2-4]. Throughout this paper the symbols $\stackrel{d}{\rightarrow}, \stackrel{P}{\rightarrow}$, and $\stackrel{\mathscr{L}^{1}}{\longrightarrow}$ stand for the convergence in distribution, convergence in probability, and convergence in $\mathscr{L}^{1}$-norm, respectively. For the convenience of the reader we repeat the relevant material from $[2,3]$ without proofs. Specifically, we have for every $X \in \mathscr{L}^{1}$ the following.

(1) The expression of the distribution $F_{X}$ of $X$ and the derivative $D_{X}^{\prime}(u)$ of the dispersion function is defined as follows:

$$
F_{X}(u)=\frac{1}{2}\left(D_{X}^{\prime}(u)+1\right), \quad \forall u \in C_{F},
$$

where $C_{F}$ is a set of continuity points of $F_{X}$.

(2) The dispersion function $D_{X}(u)$ of $X$ at a point $u \in$ $(-\infty,+\infty)$ is $\mathscr{L}^{1}$-distance between $F_{X}$ and $F_{u}$ :

$$
D_{X}(u)=\int_{-\infty}^{+\infty}\left|F_{X}(x)-F_{u}(x)\right| d x
$$

where $F_{u}$ is the distribution function of the degenerate variable at the point $u$.

(3) The equivalent formulae of (1) are

$$
D_{X}(u)=u-E(X)+2 \int_{u}^{+\infty}(x-u) d F_{X}(x)
$$

or

$$
D_{X}(u)=E(X)-u+2 \int_{-\infty}^{u}(u-x) d F_{X}(x) .
$$

On the other hand, Hung and Son in [4] have established the connections of the weak convergence of the random variables with the convergence of the dispersion functions as follows.

(4) Let $\left\{X_{n}, n \geq 1\right\}$ be a sequence of $\mathscr{L}^{1}$-random variables. If there exists $p>1$, such that

$$
\sup _{n \in \mathbb{N}} E\left|X_{n}\right|^{p}<+\infty
$$

and if

$$
X_{n} \stackrel{d}{\longrightarrow} X, \quad \text { as } n \longrightarrow \infty
$$

then $D_{X_{n}}(u) \rightarrow D_{X}(u)$, for all $u \in(-\infty,+\infty)$.
(5) Let $\left\{X_{n}, n \geq 1\right\}$ be a sequence of $\mathscr{L}^{1}$-random variables, and let $\left\{D_{n}, n \geq 1\right\}$ be the corresponding of dispersion functions. Suppose that $D_{n}(u) \rightarrow D(u)$ as $n \rightarrow \infty$, for every $u \in(-\infty,+\infty)$. Then, we have the following.

(a) $D(u)$ is a convex function on $(-\infty,+\infty)$.

(b) Let $\mathscr{H}$ be the set of all points $u \in(-\infty,+\infty)$, such that $D^{\prime}(u), D_{n}^{\prime}(u)$, and $n \in \mathbb{N}$ exist. Then $\mathscr{H}$ is a dense set in $(-\infty,+\infty)$ and

$$
\lim _{n \rightarrow \infty} D_{n}^{\prime}(u)=D^{\prime}(u), \quad \forall u \in \mathscr{H} .
$$

(c) Let $\mathscr{H}_{0}$ be the set of all points such that $D^{\prime}(u)$ exists. Then for $u \in \mathscr{H}_{0}$,

$$
\lim _{u \rightarrow-\infty} D^{\prime}(u)=-1, \quad \lim _{u \rightarrow+\infty} D^{\prime}(u)=1 .
$$

(6) Let $X, X_{1}, \ldots, X_{n} \ldots \in \mathscr{L}^{1}$, and let $D, D_{1}, \ldots, D_{n}, \ldots$ be the corresponding dispersion functions. Assume that $D_{n}(u) \rightarrow D(u)$ as $n \rightarrow \infty$, for every $u \in(-\infty$, $+\infty)$. Then,

(a)

$$
X_{n} \stackrel{d}{\longrightarrow} X, \quad \text { as } n \longrightarrow \infty
$$

(b)

$$
\lim _{n \rightarrow \infty} \sup _{u \in \mathbb{R}}\left|D_{n}(u)-D(u)\right|=0 .
$$

(7) Let $\left\{X_{n}, n \geq 1\right\}$ be a sequence of $\mathscr{L}^{1}$-random variables, and let $\left\{D_{n}(u), n \geq 1\right\}$ be the corresponding sequence of dispersion functions. If the assumption $X_{n} \stackrel{d}{\rightarrow} X$ holds and $D_{n}(u) \rightarrow D(u)$ as $n \rightarrow \infty$, for every $u \in(-\infty,+\infty)$, then there exists a distribution function $F_{X}$ such that

$$
D(u)=\int_{-\infty}^{+\infty}|x-u| d F_{X}(x)
$$

(see [4] for more details).

\section{Main Results}

In this section, all the random variables or probability distribution functions mentioned are related to $\mathscr{L}^{1}$ space.

According to the results from Muñoz-Perez and SanchezGomez [2,3], for $X, Y \in \mathscr{L}^{1}$, we say that the random variable $Y$ is at least as dispersed as $X$, denoted by $X \stackrel{d}{\leq} Y$ or $F_{X} \stackrel{d}{\leq} F_{Y}$, if

$$
D_{X-E X}(u) \leq D_{Y-E Y}(u), \quad \forall u \in(-\infty,+\infty) .
$$

It can be easily seen that a degenerate variables is the lower bound of the family of finite-mean random variables. Before stating the main results of this paper, we first study some properties of dispersive ordering. 
Lemma 1. Suppose that $X$ and $Y$ are two independent random variables. Then,

$$
\begin{aligned}
D_{X+Y}(u) & =\int_{-\infty}^{+\infty} D_{X}(u-y) d F_{Y}(y) \\
& =\int_{-\infty}^{+\infty} D_{Y}(u-x) d F_{Y}(x),
\end{aligned}
$$

where $F_{X}$ and $F_{Y}$ are distribution functions of $X$ and $Y$, respectively.

Proof. Let $F$ be the distribution function of $X+Y$; we have

$$
\begin{gathered}
D_{X+Y}(u)=\int_{-\infty}^{u}(u-x) d F(x)+\int_{u}^{+\infty}(x-u) d F(x), \\
F(x)=\int_{-\infty}^{+\infty} F_{X}(x-y) d F_{Y}(y) .
\end{gathered}
$$

Hence,

$$
\begin{aligned}
& \int_{a}^{u}(u-x) d F(x) \\
&=\lim _{n \rightarrow \infty} \sum_{k=1}^{k_{n}}\left(u-x_{n_{k}}\right) F\left[x_{n_{k}} ; x_{n_{k+1}}\right) \\
&=\lim _{n \rightarrow \infty} \int_{-\infty}^{+\infty} \sum_{k=1}^{k_{n}}\left(u-x_{n_{k}}\right) F_{X} \\
& \quad \times\left[x_{n_{k}}-y ; x_{n_{k+1}}-y\right) d F_{Y}(y) \\
&=\lim _{n \rightarrow \infty} \int_{-\infty}^{+\infty} \sum_{k=1}^{k_{n}}\left[(u-y)-\left(x_{n_{k}}-y\right)\right] F_{X} \\
& \times\left[x_{n_{k}}-y ; x_{n_{k+1}}-y\right) d F_{Y}(y),
\end{aligned}
$$

where

$$
\begin{gathered}
a=x_{n_{1}}<x_{n_{2}}<\cdots<x_{n_{k_{n}+1}}=u, \\
\left.\lim _{n \rightarrow \infty_{1 \leq k \leq k_{n}}} \sup _{1 \leq n_{n_{k+1}}}-x_{n_{k}}\right)=0 .
\end{gathered}
$$

Besides,

$$
\begin{gathered}
\sum_{k=1}^{k_{n}}\left[(u-y)-\left(x_{n_{k}}-y\right)\right] F_{X}\left[x_{n_{k}}-y ; x_{n_{k+1}}-y\right) \leq u-a, \\
\lim _{n \rightarrow \infty} \sum_{k=1}^{k_{n}}\left[(u-y)-\left(x_{n_{k}}-y\right)\right] F_{X}\left[x_{n_{k}}-y ; x_{n_{k+1}}-y\right) \\
=\int_{a-y}^{u-y}(u-y-x) d F_{X}(x) .
\end{gathered}
$$

Therefore

$$
\begin{aligned}
\lim _{n \rightarrow \infty} \int_{-\infty}^{+\infty} \sum_{k=1}^{k_{n}}\left[(u-y)-\left(x_{n_{k}}-y\right)\right] F_{X} \\
\quad \times\left[x_{n_{k}}-y ; x_{n_{k+1}}-y\right) d F_{Y}(y) \\
=\int_{-\infty}^{+\infty} \int_{a-y}^{u-y}(u-y-x) d F_{X}(x) d F_{Y}(y) .
\end{aligned}
$$

Using the results just obtained, we have

$$
\begin{aligned}
\int_{-\infty}^{u}(u-x) d F(x) \\
\quad=\int_{-\infty}^{+\infty} \int_{-\infty}^{u-y}(u-y-x) d F_{X}(x) d F_{Y}(y) .
\end{aligned}
$$

By an argument analogous to the previous one, we get

$$
\begin{aligned}
\int_{u}^{+\infty} & (x-u) d F(x) \\
& =\int_{-\infty}^{+\infty} \int_{u-y}^{+\infty}(x-(u-y)) d F_{X}(x) d F_{Y}(y) .
\end{aligned}
$$

We infer that

$$
\begin{aligned}
D_{X+Y}(u) & =\int_{-\infty}^{+\infty}|x-u| d F(x) \\
& =\int_{-\infty}^{+\infty} \int_{-\infty}^{+\infty}|x-(u-y)| d F_{X}(x) d F_{Y}(y) \\
& =\int_{-\infty}^{+\infty} D_{X}(u-y) d F_{Y}(y)
\end{aligned}
$$

This completes the proof of the lemma.

Theorem 2. Suppose that the random variables $X, Y, Z \in \mathscr{L}^{1}$. Then, we have the following.

(1) If $X \stackrel{d}{\leq} Y$ and $Y \stackrel{d}{\leq} Z$, then $X \stackrel{d}{\leq} Z$.

(2) If $X \stackrel{d}{\leq} Y$ and $Y \stackrel{d}{\leq} X$, then $Y \stackrel{d}{=} X$. That means that

$$
D_{X-E X}(u)=D_{Y-E Y}(u), \quad \forall u \in(-\infty,+\infty) .
$$

(3) If $0<\alpha<1<\beta$, then $\alpha X \stackrel{d}{\leq} X \stackrel{d}{\leq} \beta X$.

(4) Let $X, Y$, and $Z$ be independent random variables in $\mathscr{L}^{1}$ and $X \stackrel{d}{\leq} Y$. Then,

$$
X+Z \stackrel{d}{\leq} Y+Z
$$

Proof. Properties (1) and (2) are obvious.

For the property (3), suppose that $E(X)=0$. We will show that $\alpha X \stackrel{d}{\leq} X$, since the rest is equivalent to this. 
Note that

$$
D_{\alpha X}(u)=E|\alpha X-u|=\alpha E\left|X-\frac{1}{\alpha} u\right|=\alpha D_{X}\left(\frac{1}{\alpha} u\right) .
$$

According to that, the dispersion function is a convex function whose derivative exists almost everywhere and is bounded by -1 and 1 . Hence,

$$
-1 \leq \frac{D_{X}(u)-D_{X}((1 / \alpha) u)}{u-(1 / \alpha) u} \leq 1, \quad \forall u \neq 0 .
$$

This gives

$$
\begin{array}{ll}
\alpha D_{X}(u)+u-\alpha u \geq \alpha D_{X}\left(\frac{1}{\alpha} u\right), & \forall u>0, \\
\alpha D_{X}(u)-u+\alpha u \geq \alpha D_{X}\left(\frac{1}{\alpha} u\right), & \forall u<0 .
\end{array}
$$

Besides,

$$
\begin{aligned}
D_{X}(u)= & E|X-u|=u-E(X)+2 \int_{u}^{+\infty}(x-u) d F_{X}(x) \\
D_{X}(u) & =E|X-u| \\
& =E(X)-u+2 \int_{-\infty}^{u}(u-x) d F_{X}(x) \geq|u|
\end{aligned}
$$

Combining the (27) and (28) or (29), we get the complete proof.

The last property (4) can be obtained from the previous lemma.

The following theorem gives us an important property of dispersive ordering.

Theorem 3. Let $\left(F_{n}\right)_{n \in \mathbb{N}}$ be a sequence of distribution functions. If they are monotone and bounded in the meaning of dispersive ordering, then they converge weakly.

Proof. Let $\left(D_{n}\right)_{n \in \mathbb{N}}$ be the corresponding dispersion functions of $\left(F_{n}\right)_{n \in \mathbb{N}}$. Since $\left(F_{n}\right)_{n \in \mathbb{N}}$ is monotone and bounded, the sequence $\left(D_{n}(u)\right)_{n \in \mathbb{N}}$ is monotone and bounded for each $u \in$ $(-\infty,+\infty)$ as well. That means that there exists $D(u)$ such that $D_{n}(u) \rightarrow D(u)$ for every $u \in(-\infty,+\infty)$.

According to the properties of the dispersion functions shown in Section 2, we get the complete proof.

From the Theorem 3, we have the following interesting results.

Corollary 4. If $\left\{X_{n}, \mathscr{F}_{n}\right\}$ is a martingale and $\mathscr{L}^{1}$-bounded, then the corresponding sequence of distribution functions converges weakly.

Proof. It is know that if $\left\{X_{n}, \mathscr{F}_{n}\right\}$ is a martingale, then $\left(E \mid X_{n}-\right.$ $u \mid)_{n}$ is a nondecreasing sequence. From the bounded condition and Theorem 3, we obtain the conclusion.
Theorem 5. Let $\left\{X_{n}, n \geq 1\right\}$ be a sequence of $\mathscr{L}^{1}$-independent random variables. Moreover, suppose that $X$ is a $\mathscr{L}^{1}$-random variable $X$, satisfying

$$
X_{n} \stackrel{d}{\leq} X, \quad \forall n \in \mathbb{N}
$$

Then

$$
\frac{S_{n}-E\left(S_{n}\right)}{n} \stackrel{\mathscr{L}^{1}}{\longrightarrow} 0,
$$

where $S_{n}=X_{1}+X_{2}+\cdots+X_{n}$.

Proof. Without loss of generality, we assume that $E(X)=$ $E\left(X_{n}\right)=0$, for all $n \geq 1$.

Suppose that $\left(Y_{n}\right)_{n}$ is a sequence of independent random variables such that $P\left(Y_{n}<x\right)=P(X<x)=F(x)$, for all $n \in \mathbb{N}, x \in(-\infty,+\infty)$.

We have

$$
\begin{aligned}
D_{\left(Y_{1}+Y_{2}+\cdots+Y_{n}\right) / n}(u) & =\frac{1}{n} D_{Y_{1}+Y_{2}+\cdots+Y_{n}}(n u) \\
& =\frac{1}{n} \int_{-\infty}^{+\infty}|x-n u| d F^{n *}(x),
\end{aligned}
$$

where $*$ is the notation of convolution between distribution functions and

$$
F^{n *}=F * F * \cdots * F(n \text { times }) .
$$

Note that

$$
\begin{gathered}
D_{\left(Y_{1}+Y_{2}+\cdots+Y_{n}\right) / n}(u) \geq|u|, \\
\frac{1}{n} D_{Y_{1}+Y_{2}+\cdots+Y_{n}}(n u) \leq E\left|\frac{Y_{1}+Y_{2}+\cdots+Y_{n}}{n}\right|+|u|, \\
\lim _{n \rightarrow \infty} E\left|\frac{Y_{1}+Y_{2}+\cdots+Y_{n}}{n}\right|=0 .
\end{gathered}
$$

We obtain

$$
\lim _{n \rightarrow \infty} D_{\left(Y_{1}+Y_{2}+\cdots+Y_{n}\right) / n}(u)=|u| .
$$

Since $X_{n} \stackrel{d}{\leq} X$, we have

$$
S_{n} \stackrel{d}{\leq} Y_{1}+Y_{2}+\cdots+Y_{n}
$$

This means that

$$
D_{S_{n} / n}(u) \leq D_{\left(Y_{1}+Y_{2}+\cdots+Y_{n}\right) / n}(u) .
$$

It is implied from (35) and (37) that

$$
\lim D_{S_{n} / n}(u)=|u| .
$$

According to the previous proposition,

$|u| \leq D_{\left(S_{n}-E\left(S_{n}\right)\right) / n}(u) \leq \frac{1}{n} \int_{-\infty}^{+\infty}|x-n E(X)-u| d F_{X}^{n *}(x)$. 
Otherwise,

$$
\lim _{n \rightarrow \infty} \frac{1}{n} \int_{-\infty}^{+\infty}|x-n E(X)-u| d F^{n *}(x)=|u| .
$$

Hence,

$$
\lim _{n \rightarrow \infty} D_{\left(S_{n}-E\left(S_{n}\right)\right) / n}(u)=|u| .
$$

And this completes the proof.

Note that it makes sense to consider that the dispersive ordering can be applied to limit theorems in probability (for the $\mathscr{L}^{1}$-weak law of large numbers) as a new approach and should be more investigated.

Moreover, as we know that a famous estimator is minimum-variance unbiased one. This is based on the existence of variance, which is considered as a measure of dispersion. It is natural to link to an estimator based on dispersive ordering.

Definition 6. Suppose that $\left(X_{1}, X_{2}, \ldots, X_{n}\right)$ is a random sample from the family of distribution $F(x, \theta)$ and the estimator $\widehat{\theta}$ is called a minimum-dispersive unbiased estimator if $\widehat{\theta}$ is unbiased and holds $\widehat{\theta} \stackrel{d}{\leq} \theta^{*}$ for every unbiased estimator $\theta^{*}$ of $\theta$.

It can be shown that the sample mean is a good estimator in the meaning of dispersive ordering.

Proposition 7. If $\left(X_{1}, X_{2}, \ldots, X_{n}\right)$ is a random sample from the family of distribution $F(x, \theta)$ with finite mean, then

$$
\frac{X_{1}+X_{2}+\cdots+X_{n}}{n} \stackrel{d}{\leq} \alpha_{1} X_{1}+\alpha_{2} X_{2}+\cdots+\alpha_{n} X_{n},
$$

where $\alpha_{i}>0, \alpha_{1}+\alpha_{2}+\cdots+\alpha_{n}=1$.

Proof. Since $\alpha_{1} X_{1}+\alpha_{2} X_{2}+\cdots+\alpha_{n} X_{n}, \alpha_{2} X_{1}+\alpha_{3} X_{2}+\cdots+\alpha_{1} X_{n}$, $\ldots, \alpha_{n} X_{1}+\alpha_{1} X_{2}+\cdots+\alpha_{n-1} X_{n}$ have the same distribution, we have

$$
\begin{aligned}
n E\left|\alpha_{1} X_{1}+\alpha_{2} X_{2}+\cdots+\alpha_{n} X_{n}-u\right| \\
=E\left|\alpha_{1} X_{1}+\alpha_{2} X_{2}+\cdots+\alpha_{n} X_{n}-u\right| \\
\quad+\cdots+E\left|\alpha_{n} X_{1}+\alpha_{1} X_{2}+\cdots+\alpha_{n-1} X_{n}-u\right| \\
\geq E \mid\left(\alpha_{1}+\alpha_{2}+\cdots+\alpha_{n}\right) X_{1}+\cdots \\
\quad+\left(\alpha_{1}+\alpha_{2}+\cdots+\alpha_{n}\right) X_{n}-n u \mid,
\end{aligned}
$$

and this implies the proof.

Proposition 8. If

$$
D_{\widehat{\theta}_{n}-\theta}(u) \longrightarrow D_{0}(u), \quad \text { as } n \longrightarrow \infty, \forall u \in \mathbb{R},
$$

then $\hat{\theta}_{n}$ is a consistent estimator of $\theta$, where $D_{0}$ is the dispersion function of degenerated random variable at 0 .

Proof. According to the properties of dispersion functions, we have $\widehat{\theta}_{n}-\theta \stackrel{d}{\rightarrow} 0$, and it follows that

$$
\widehat{\theta}_{n}-\theta \stackrel{P}{\longrightarrow} 0, \quad \text { as } n \longrightarrow \infty \text {. }
$$

The proof is completed.

\section{Acknowledgments}

The authors wish to express their gratitude to the referees for valuable remarks and comments improving the previous version of this paper. This work is supported by Vietnam's National Foundation For Science and Technology Development (NAFOSTED, Vietnam), Grant 101.01-2010.02.

\section{References}

[1] T. Pham-Gia and T. L. Hung, "Bayesian estimation under estimation constraint," Acta Mathematica Vietnamica, vol. 28, no. 2, pp. 201-207, 2003.

[2] J. Muñoz-Perez and A. Sanchez-Gomez, "Dispersive ordering by dilation," Journal of Applied Probability, vol. 27, no. 2, pp. 440-444, 1990.

[3] J. Muñoz-Perez and A. Sanchez-Gomez, "A characterization of the distribution function: the dispersion function," Statistics and Probability Letters, vol. 10, no. 3, pp. 235-239, 1990.

[4] T. L. Hung and N. V. Son, "Some connections of weak convergence with the convergence of the dispersion functions," Vietnam Journal of Mathematics, vol. 31, no. 3, pp. 325-332, 2003.

[5] J. Bartoszewicz, "Dispersive functions and stochastic orders," Applicationes Mathematicae, vol. 24, no. 4, pp. 429-444, 1997.

[6] M. Shaked and J. G. Shanthikumar, Stochastic Orders, Springer Series in Statistics, Springer, New York, NY, USA, 2007.

[7] T. Pham-Gia, N. Turkkan, and Q. P. Duong, "Using the mean deviation in the elicitation of the prior distribution," Statistics \& Probability Letters, vol. 13, no. 5, pp. 373-381, 1992.

[8] T. Pham-Gia and T. L. Hung, "The mean and median absolute deviations," Mathematical and Computer Modelling, vol. 34, no. 7-8, pp. 921-936, 2001.

[9] T. L. Hung and T. Pham-Gia, "On the mean absolute deviation of the random variables," Vietnam National University, Journal of Science, vol. 15, pp. 36-44, 1999. 


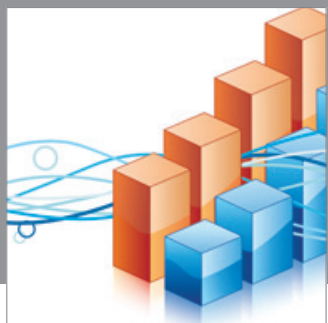

Advances in

Operations Research

mansans

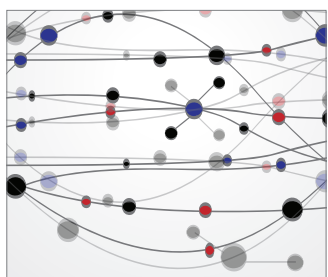

The Scientific World Journal
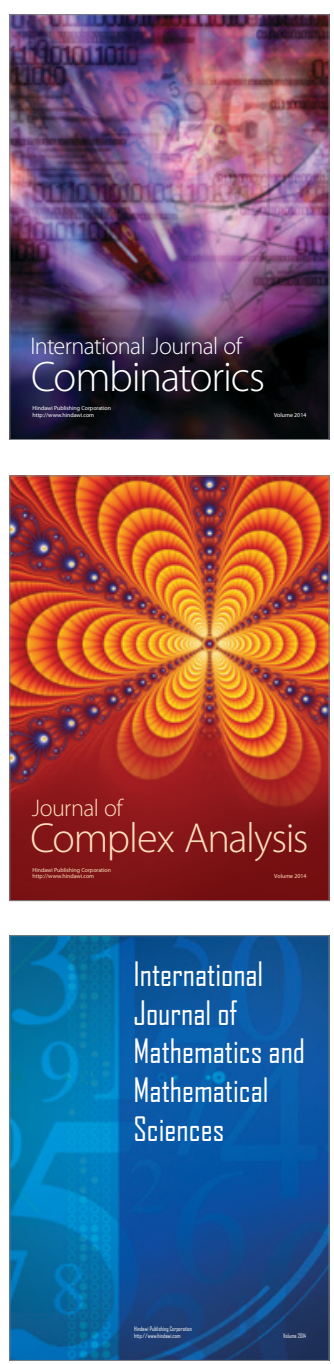
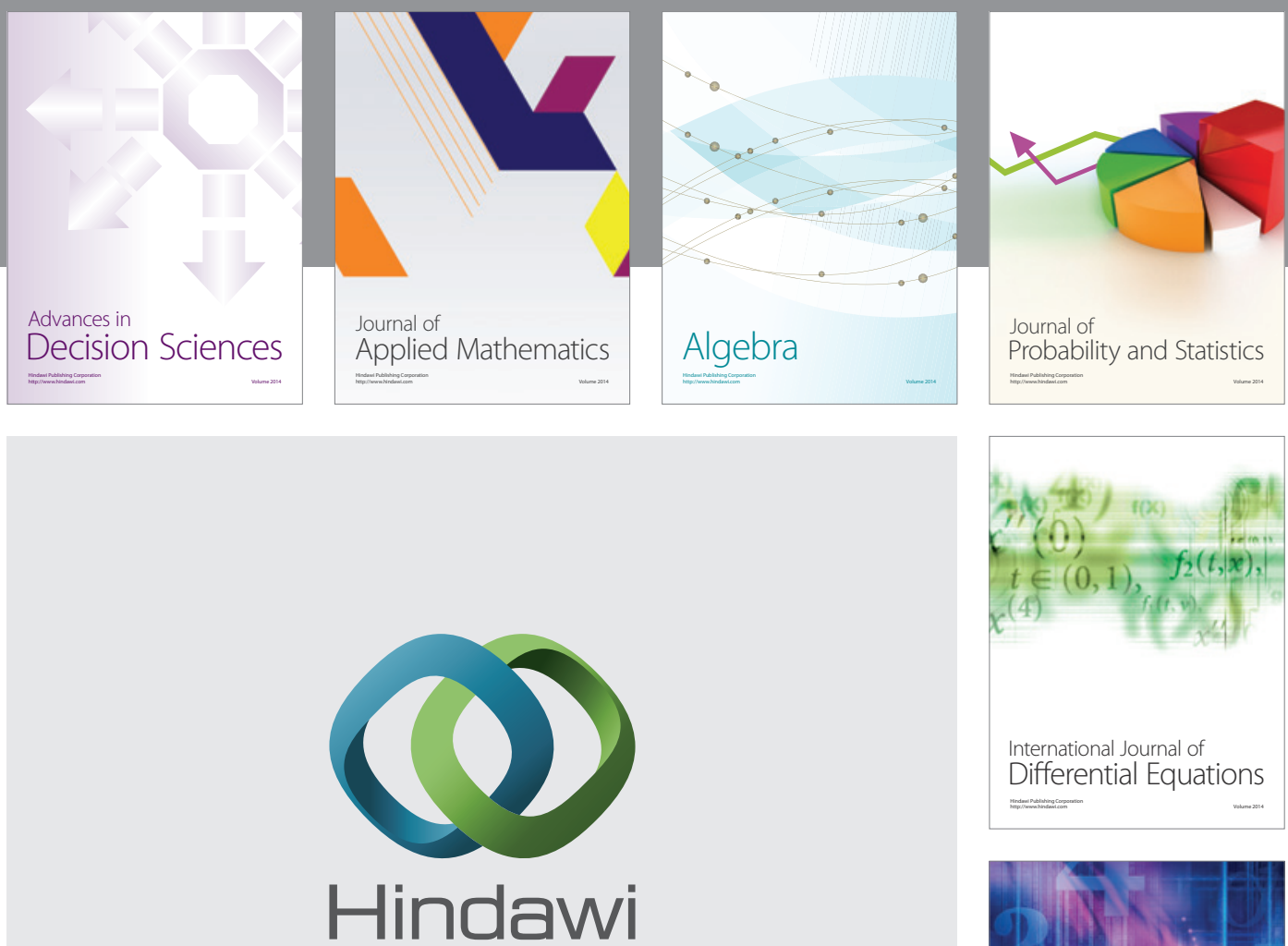

Submit your manuscripts at http://www.hindawi.com
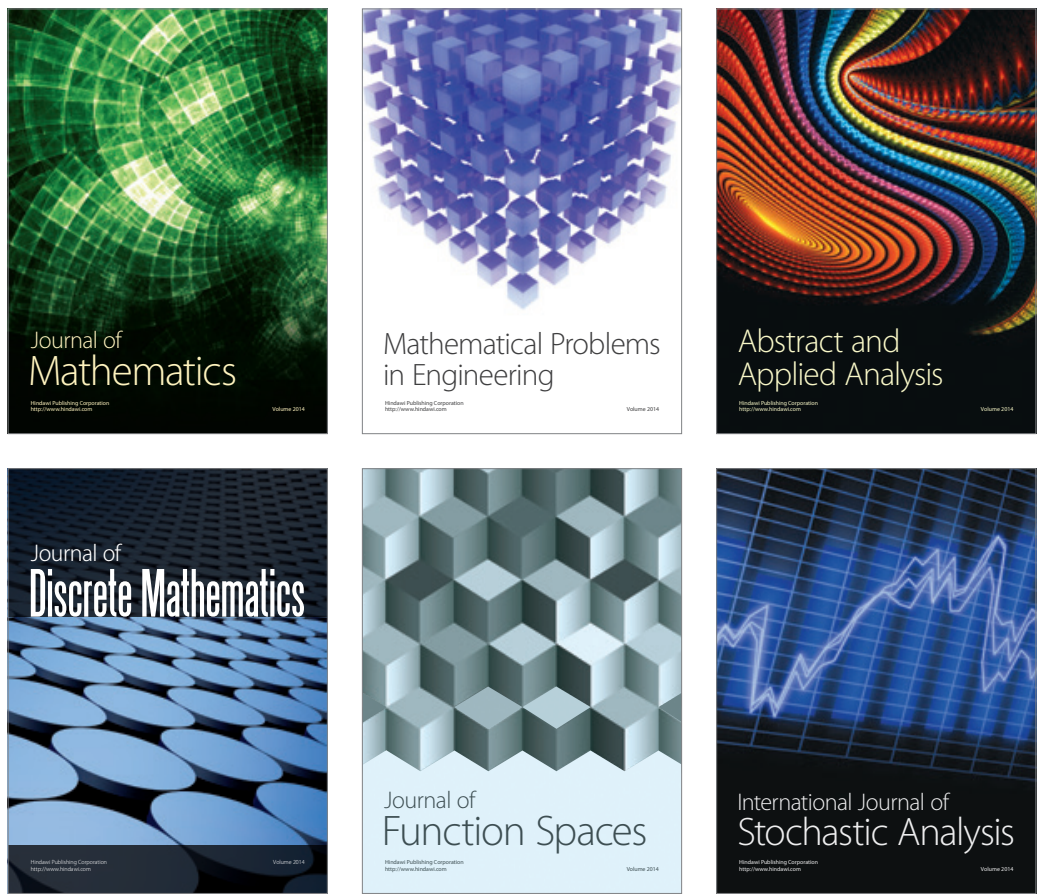

Journal of

Function Spaces

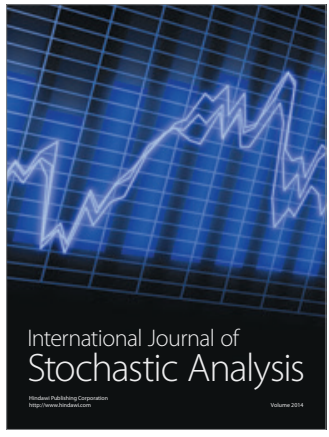

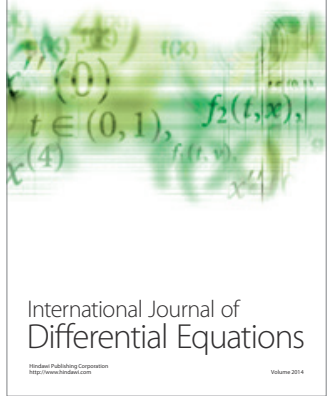
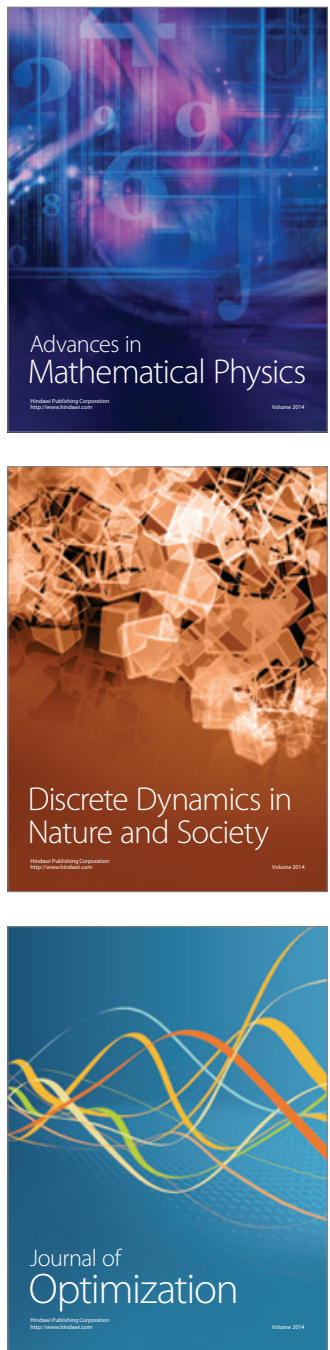